\title{
Computational Modelling of Cholera Bacteriophage with Treatment
}

\author{
Daniel S. Mgonja ${ }^{1}$, Estomih S. Massawe ${ }^{1}$, Oluwole Daniel Makinde ${ }^{2}$ \\ ${ }^{1}$ Mathematics Department, University of Dar es Salaam, Dar es Salaam, Tanzania \\ ${ }^{2}$ Faculty of Military Science, Stellenbosch University, Stellenbosch, South Africa \\ Email: mgonja d@yahoo.com
}

Received 17 June 2015; accepted 31 July 2015; published 3 August 2015

Copyright (C) 2015 by authors and Scientific Research Publishing Inc.

This work is licensed under the Creative Commons Attribution International License (CC BY).

http://creativecommons.org/licenses/by/4.0/

c) (i) Open Access

\section{Abstract}

This paper examines the computational modelling of cholera bacteriophage with treatment. A nonlinear mathematical model for cholera bacteriophage and treatment is formulated and analysed. The effective reproduction number of the nonlinear model system is calculated by next generation operator method. By using the next generation matrix approach, the disease-free equilibrium is found to be locally stable at threshold parameter less than unity and unstable at threshold parameter greater than unity. Globally, the disease free equilibrium point is not stable due to existence of forward bifurcation at threshold parameter equal to unity. Stability analysis and numerical simulations suggest that the combination of bacteriophage and treatment may contribute to lessening the severity of cholera epidemics by reducing the number of Vibrio cholerae in the environment. Hence with the presence of bacteriophage virus and treatment, cholera is self-limiting in nature.

\section{Keywords}

Cholera, Bacteriophage, Treatment, Vibrio cholerae, Equilibrium, Stability, Effective Reproduction Number

\section{Introduction}

A highly pathogenic gram-negative bacterium Vibrio cholerae is the causative agent of the water-born diarrheal disease; cholera [1]. The sensitive diarrheal infection is caused by ingestion of food or water contaminated with the bacterium Vibrio cholerae. The most common symptoms of cholera include severe watery diarrhea, vomiting, excessive thirst, loss of skin elasticity and muscle cramps [2]. The most important treatment is to replace the

*Corresponding author. 
fluids and electrolytes that have been lost due to diarrhea [3]. This is done either through oral fluid rehydration or, in severe cases, intravenous fluid rehydration.

Treatment of cholera with massive doses of bacteriophage is not as effective as treatment with tetracycline. However, bacteriophage can selectively eliminate the majority of Vibrios without affecting the other intestinal flora toxic effect on the patient.

Bacteriophage might be useful as a research tool [4] [5] developed a model which accounted for the role played by Hiperinfectivity Vibrios in causing the cholera epidemic. A mathematical model by [6] was analysed to study the dynamics of cholera in terms of interaction between Vibrio cholerae and bacteriophage model.. However, in all the above studies, none of them incorporated the treatment. In this paper, it is therefore intended to analyse a model which incorporates the treatment. We thus study and analyse a nonlinear mathematical model of the cholera bacteriophage and treatment. The model incorporates the assumption that there is natural death of human, Vibrio cholerae and bacteriophage at the rates of $\mu_{1}, \mu_{2}$ and $\mu_{3}$.

\section{Model Formulation}

A nonlinear mathematical model is proposed and analysed to study the impact of bacteriophage and treatment in the environment while the cholera epidemic is in progress. The proposed mathematical model divides the human population, $N(t)$ into four classes at time $t$, namely; the susceptible population $S(t)$, infected population $I(t)$, the treated population $T(t)$, and the recovery population $R(t)$. The aquatic population of bacteria $V(t)$, denotes the concentration of toxigenic Vibrio cholerae in water, and $P(t)$, represent the phage density at any time $t$.

A Susceptible, Infective, Treatment, Recovery and Susceptible (SITRS) model is developed to study the role of bacteriophage and treatment in the environment during the cholera outbreaks. The SITRS model indicates that the passage of individual is from the susceptible class $S(t)$, Infective class $I(t)$, treatment class $T(t)$, recovery class $R(t)$ and then becomes susceptible again. In this model, it is assumed that the susceptible people are recruited in the population at a constant immigration rate $a$.

In formulating the model, the following assumptions are taken in consideration:

1) The population varies.

2) There is natural death of human, Vibrio cholerae and bacteriophage at the rates of $\mu_{1} \mu_{2}$ and $\mu_{3}$.

3) The disease is fatal.

4) The rate of transmission is directly proportional to the susceptible population and also to the ratio between the members of infected population to the environment.

5) The population is homogeneously mixed and each susceptible individual has equal chance of acquiring cholera.

However, the model also assumes that the infected individual can recover at the rate of $\tau$ and some susceptible individuals acquire cholera infection following contacts with the pathogenic Vibrio cholerae at the rate of $\lambda$ given by $\lambda=\frac{\alpha V}{\kappa+V}$ where $\alpha$ is the rate of contact to contaminated water per unity time, $\kappa$ is the concentration of Vibrio cholerae in water that yields $50 \%$ chance of spreading cholera, and $D(V)=\frac{V}{\kappa+V}$ is the probability that an individual in contact with untreated water with pathogenic Vibrios is infected with Vibrio cholerae.

Taking into account the above considerations and assumptions then we have the following schematic flow diagram (Figure 1):

The model is thus governed by the following system of nonlinear ordinary differential equations:

$$
\begin{gathered}
\frac{\mathrm{d} S}{\mathrm{~d} t}=a-\frac{\alpha V S}{\kappa+V}+\phi R-\mu_{1} S, \\
\frac{\mathrm{d} I}{\mathrm{~d} t}=\frac{\alpha V S}{\kappa+V}-\left(\tau+\varepsilon+\mu_{1}+\sigma\right) I, \\
\frac{\mathrm{d} V}{\mathrm{~d} t}=g+\delta I-\mu_{2} V-\gamma V,
\end{gathered}
$$




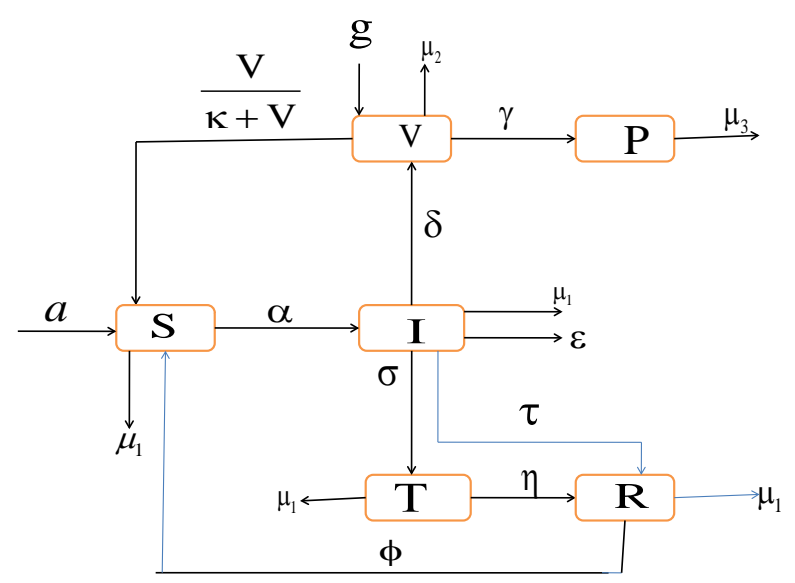

Figure 1. A compartment model for Cholera Bacteriophage with treatment [6].

$$
\begin{gathered}
\frac{\mathrm{d} P}{\mathrm{~d} t}=\gamma V-\mu_{3} P, \\
\frac{\mathrm{d} T}{\mathrm{~d} t}=\sigma I-\left(\mu_{1}+\eta\right) T, \\
\frac{\mathrm{d} R}{\mathrm{~d} t}=\eta T-\left(\mu_{1}+\phi\right) R+\tau I,
\end{gathered}
$$

where

$$
S(0) \geq 0, I(0) \geq 0, T(0) \geq 0, R(0) \geq 0, T(0) \geq 0, P(0) \geq 0 .
$$

The total population of human at time $t$ is given by

$$
N_{H}=S(t)+I(t)+T(t)+R(t)
$$

Thus it follows that

$$
\frac{\mathrm{d} N_{H}}{\mathrm{~d} t}=\frac{\mathrm{d} S}{\mathrm{~d} t}+\frac{\mathrm{d} I}{\mathrm{~d} t}+\frac{\mathrm{d} T}{\mathrm{~d} t}+\frac{\mathrm{d} R}{\mathrm{~d} t}
$$

This implies that

$$
\begin{aligned}
\frac{\mathrm{d} N_{H}}{\mathrm{~d} t}= & a-\frac{\alpha V S}{\kappa+V}-\mu_{1} S+\phi R+\frac{\alpha V S}{\kappa+V}-\left(\tau+\varepsilon+\mu_{1}+\sigma\right) I \\
& +\sigma I-\left(\mu_{1}+\eta\right) T+\eta T-\left(\mu_{1}+\phi\right) R+\tau I
\end{aligned}
$$

This reduces to

$$
\frac{\mathrm{d} N_{H}}{\mathrm{~d} t}=a-\mu_{1} N_{H}-\varepsilon I
$$

It follows that from equation (2), that in the absence of the disease i.e. $\varepsilon=0$, the rate of change of human population size is given by

$$
\frac{\mathrm{d} N_{H}}{\mathrm{~d} t}=a-N_{H} \mu_{1} .
$$

\section{Model Analysis}

The model system of Equations (1) will be analysed qualitatively to get insight into its dynamical features which 
will give a better understanding of the effects of bacteriophage and treatment while cholera epidemic persist in a given population. The effective reproductive number $R_{e}$ which governs elimination or persistence of cholera will be determined and studied.

\subsection{Disease Free Equilibrium (DFE)}

The disease free equilibrium of the model system of equations (1) is obtained by setting

$$
\frac{\mathrm{d} S}{\mathrm{~d} t}=\frac{\mathrm{d} I}{\mathrm{~d} t}=\frac{\mathrm{d} T}{\mathrm{~d} t}=\frac{\mathrm{d} R}{\mathrm{~d} t}=\frac{\mathrm{d} V}{\mathrm{~d} t}=\frac{\mathrm{d} P}{\mathrm{~d} t}=0
$$

At disease-free equilibrium, we have $I=0, T=0, V=0, R=0, P=0, g=0$. consequently we get

$$
S=\frac{a}{\mu_{1}} .
$$

Therefore the disease free equilibrium (DFE) denoted by $E_{0}$ of the cholera bacteriophage and treatment model system (1) is given by

$$
E_{0}=(S, 0,0,0,0,0)=\left(\frac{a}{\mu_{1}}, 0,0,0,0,0\right)
$$

\subsection{The Effective Reproduction Number, " $R$ "}

The effective reproduction number, $R_{e}$ of the nonlinear model system (1) was obtained by using the next generation operator method and is given by

$$
R_{e}=\frac{\alpha \delta a}{\kappa \mu_{1}\left(\tau+\varepsilon+\mu_{1}+\sigma\right)\left(\mu_{2}+\gamma\right)}
$$

\subsection{Local Stability of Disease Free Equilibrium Point}

The disease-free equilibrium of the nonlinear model system (1) is given by

$$
E_{0}=(S, 0,0,0,0,0)=\left(\frac{a}{\mu_{1}}, 0,0,0,0,0\right)
$$

\section{Theorem 1}

The local stability of the disease-free equilibrium of the cholera Bacteriophage and treatment model system (1) is locally asymptotically stable if $R_{e}<1$ and unstable if $R_{e}>1$.

This is shown by computing the Jacobian matrix of the model (1). The Jacobian matrix is computed by differentiating each equation in the system with respect to the state variables $S, I, V, P, T, R$.

It follows that

$$
\boldsymbol{J}=\left(\begin{array}{llllll}
\frac{\partial f_{1}}{\partial S}\left(E_{0}\right) & \frac{\partial f_{1}}{\partial I}\left(E_{0}\right) & \frac{\partial f_{1}}{\partial V}\left(E_{0}\right) & \frac{\partial f_{1}}{\partial P}\left(E_{0}\right) & \frac{\partial f_{1}}{\partial T}\left(E_{0}\right) & \frac{\partial f_{1}}{\partial R}\left(E_{0}\right) \\
\frac{\partial f_{2}}{\partial S}\left(E_{0}\right) & \frac{\partial f_{2}}{\partial I}\left(E_{0}\right) & \frac{\partial f_{2}}{\partial V}\left(E_{0}\right) & \frac{\partial f_{2}}{\partial P}\left(E_{0}\right) & \frac{\partial f_{2}}{\partial T}\left(E_{0}\right) & \frac{\partial f_{2}}{\partial R}\left(E_{0}\right) \\
\frac{\partial f_{3}}{\partial S}\left(E_{0}\right) & \frac{\partial f_{3}}{\partial I}\left(E_{0}\right) & \frac{\partial f_{3}}{\partial V}\left(E_{0}\right) & \frac{\partial f_{3}}{\partial P}\left(E_{0}\right) & \frac{\partial f_{3}}{\partial T}\left(E_{0}\right) & \frac{\partial f_{3}}{\partial R}\left(E_{0}\right) \\
\frac{\partial f_{4}}{\partial S}\left(E_{0}\right) & \frac{\partial f_{4}}{\partial I}\left(E_{0}\right) & \frac{\partial f_{4}}{\partial V}\left(E_{0}\right) & \frac{\partial f_{4}}{\partial P}\left(E_{0}\right) & \frac{\partial f_{4}}{\partial T}\left(E_{0}\right) & \frac{\partial f_{4}}{\partial R}\left(E_{0}\right) \\
\frac{\partial f_{5}}{\partial S}\left(E_{0}\right) & \frac{\partial f_{5}}{\partial I}\left(E_{0}\right) & \frac{\partial f_{5}}{\partial V}\left(E_{0}\right) & \frac{\partial f_{5}}{\partial P}\left(E_{0}\right) & \frac{\partial f_{5}}{\partial T}\left(E_{0}\right) & \frac{\partial f_{5}}{\partial R}\left(E_{0}\right) \\
\frac{\partial f_{6}}{\partial S}\left(E_{0}\right) & \frac{\partial f_{6}}{\partial I}\left(E_{0}\right) & \frac{\partial f_{6}}{\partial V}\left(E_{0}\right) & \frac{\partial f_{6}}{\partial P}\left(E_{0}\right) & \frac{\partial f_{6}}{\partial T}\left(E_{0}\right) & \frac{\partial f_{6}}{\partial R}\left(E_{0}\right)
\end{array}\right)
$$


This gives

$$
\boldsymbol{J}_{E_{0}}=\left[\begin{array}{cccccc}
-\mu_{2} & 0 & -\frac{a \alpha}{\mu_{1} \kappa} & 0 & 0 & \phi \\
0 & -\left(\tau+\varepsilon+\sigma+\mu_{1}\right) & \frac{a \alpha}{\mu_{1} \kappa} & 0 & 0 & 0 \\
0 & \delta & -\left(\gamma+\mu_{2}\right) & 0 & 0 & 0 \\
0 & 0 & \gamma & -\mu_{3} & 0 & 0 \\
0 & \sigma & 0 & 0 & -\left(\eta+\mu_{1}\right) & 0 \\
0 & \tau & 0 & 0 & \eta & -\left(\phi+\mu_{1}\right)
\end{array}\right]
$$

The characteristic equation corresponding to $\boldsymbol{J}_{E_{0}}$ is

$$
-\left[\frac{\left(-\eta-\lambda-\mu_{1}\right)\left(-\lambda-\phi-\mu_{1}\right)\left(\lambda+\mu_{1}\right)\left(-a \alpha \delta-\kappa \mu_{1}\left(\tau+\varepsilon+\lambda+\sigma+\mu_{1}\right)\left(-\gamma-\lambda-\mu_{2}\right)\right)\left(-\lambda-\mu_{3}\right)}{\kappa \mu_{1}}\right]=0
$$

It follows that

$$
\begin{gathered}
\lambda_{1}=-\mu_{1} \\
\lambda_{2}=-\left[\frac{\kappa \mu_{1}\left(\tau+\gamma+\epsilon+\sigma+\mu_{1}+\mu_{2}\right)-\sqrt{\kappa \mu_{1}\left(4 a \alpha \delta+\kappa \mu_{1}\left(\tau-\gamma+\epsilon+\sigma+\mu_{1}-\mu_{2}\right)^{2}\right)}}{2 \kappa \mu_{1}}\right]
\end{gathered}
$$

when $\sqrt{\kappa \mu_{1}\left(4 a \alpha \delta+\kappa \mu_{1}\left(\tau-\gamma+\epsilon+\sigma+\mu_{1}-\mu_{2}\right)^{2}\right)}$ is not a real number,

$$
\lambda_{3}=-\left[\frac{-\kappa \mu_{1}\left(\tau+\gamma+\epsilon+\sigma+\mu_{1}+\mu_{2}\right)+\sqrt{\kappa \mu_{1}\left(4 a \alpha \delta+\kappa \mu_{1}\left(\tau-\gamma+\epsilon+\sigma+\mu_{1}-\mu_{2}\right)^{2}\right)}}{2 \kappa \mu_{1}}\right]
$$

when $\kappa \mu_{1}\left(\tau+\gamma+\epsilon+\sigma+\mu_{1}+\mu_{2}\right)<0$ and $\sqrt{-\kappa \mu_{1}\left(4 a \alpha \delta+\kappa \mu_{1}\left(\tau-\gamma+\epsilon+\sigma+\mu_{1}-\mu_{2}\right)^{2}\right)}$ is not a real number.

Other eigenvalues are

$$
\begin{aligned}
& \lambda_{4}=-\mu_{3}, \\
& \lambda_{5}=-\left(\eta+\mu_{1}\right), \lambda_{6}=-\left(\phi+\mu_{1}\right) .
\end{aligned}
$$

Since all Eigen values of the characteristic equation have negative real parts then the disease-free equilibrium $E_{0}$ is locally asymptotically stable.

\subsection{Global Stability of Disease Free Equilibrium $E_{0}$}

Theorem 2

The disease-free equilibrium point $E_{0}$ is globally asymptotically stable if $R_{e}<1$ when all solutions of system (1) which starts in $R_{+}^{6}$ are bounded.

Proof

From system (1) we have

$$
\left(\begin{array}{c}
\frac{\mathrm{d} I}{\mathrm{~d} t} \\
\frac{\mathrm{d} V}{\mathrm{~d} t}
\end{array}\right)=(\boldsymbol{F}-\boldsymbol{V})\left(\begin{array}{c}
I \\
V
\end{array}\right)-\left(\begin{array}{cc}
0 & \frac{a \alpha}{\kappa \mu_{1}}-\frac{\alpha S}{\kappa+V} \\
0 & 0
\end{array}\right)\left(\begin{array}{c}
I \\
V
\end{array}\right)
$$


where the matrices $\boldsymbol{F}$ and $\boldsymbol{V}$ are given by $\boldsymbol{F}=\left[\begin{array}{cc}0 & \frac{\alpha a}{\kappa \mu_{1}} \\ 0 & 0\end{array}\right]$ and $\boldsymbol{V}=\left[\begin{array}{cc}\left(\tau+\varepsilon+\mu_{1}+\sigma\right) & 0 \\ -\delta & \mu_{2}+\gamma\end{array}\right]$. Since $\frac{a}{\mu_{1}}>\frac{\kappa S}{\kappa+V} \quad \forall t \geq 0$ in $R_{+}^{6}$, then

$$
\left(\begin{array}{c}
\frac{\mathrm{d} I}{\mathrm{~d} t} \\
\frac{\mathrm{d} V}{\mathrm{~d} t}
\end{array}\right) \leq(\boldsymbol{F}-\boldsymbol{V})\left(\begin{array}{c}
I \\
V
\end{array}\right)
$$

Using the fact that the eigenvalues of the matrix $(\boldsymbol{F}-\boldsymbol{V})$ all have negative real parts it follows that the linearized differential inequality system (1) is stable whenever $R_{e}<1$ [7]. Consequently, $I(t), V(t) \rightarrow(0,0)$ as $t \rightarrow \infty$. It follows by comparison theorem [8] that $I(t), V(t) \rightarrow(0,0)$. Substituting $I=V=R=0$ in the first and fourth equations of the model system (1), we obtain $S(t) \rightarrow \frac{a}{\mu_{1}}$ and $P(t) \rightarrow 0$ as $t \rightarrow \infty$. Thus $S(t), I(t), V(t), P(t), R(t), T(t) \rightarrow\left(\frac{a}{\mu_{1}}, 0,0,0,0,0\right)$ as $t \rightarrow \infty$ if $R_{e}<1$, and subsequently $E_{0}$ is globally asymptotically stable if $R_{e}<1$.

\subsection{The Endemic Equilibrium point (EEP) and Local Stability}

The endemic equilibrium of the nonlinear model system (1) is given by

$E^{*}\left(S^{*}, I^{*}, V^{*}, P^{*}, T^{*}, R^{*}\right)$. It is obtained by setting the right hand side of each equation of the nonlinear model system (1) equal to zero for $R_{e}>1 . S^{*}, I^{*}, V^{*}, P^{*}, T^{*}$ and $R^{*}$ satisfies the following relations:

$$
\begin{aligned}
& S^{*}=\frac{a}{\mu_{1}+\lambda^{*}\left(1-\frac{\tau+\frac{\eta \sigma \phi}{\left(\eta+\mu_{1}\right)\left(\phi+\mu_{1}\right)}}{\tau+\delta+\sigma+\mu_{1}}\right)} \\
& I^{*}=\frac{\lambda^{*} a}{\left(\tau+\varepsilon+\mu_{1}+\sigma\right)\left(\mu_{1}+\lambda^{*}\right)\left[1-\frac{\tau+\frac{\eta \sigma \phi}{\left(\eta+\mu_{1}\right)\left(\phi+\mu_{1}\right)}}{\tau+\varepsilon+\mu_{1}+\sigma}\right]} \\
& V^{*}=\frac{\lambda^{*} a \delta}{\left(\mu_{2}+\gamma\right)\left(\tau+\varepsilon+\mu_{1}+\sigma\right)\left(\mu_{1}+\lambda^{*}\right)\left[1-\frac{\tau+\frac{\eta \sigma \phi}{\left(\eta+\mu_{1}\right)\left(\phi+\mu_{1}\right)}}{\tau+\varepsilon+\mu_{1}+\sigma}\right]} \\
& P^{*}=\frac{\gamma \lambda^{*} \delta a}{\mu_{3}\left(\tau+\varepsilon+\mu_{1}+\sigma\right)\left(\mu_{2}+\gamma\right)\left(\mu_{1}+\lambda^{*}\left(1-\frac{\tau+\frac{\eta \sigma \phi}{\left(\eta+\mu_{1}\right)\left(\phi+\mu_{1}\right)}}{\left(\tau+\varepsilon+\sigma+\mu_{1}\right)}\right)\right.}
\end{aligned}
$$




$$
\begin{aligned}
& T^{*}=\frac{\sigma \lambda^{*} a}{\left(\mu_{1}+\eta\right)\left(\tau+\varepsilon+\mu_{1}+\sigma\right)\left(\mu_{1}+\lambda^{*}\left(1-\frac{\tau+\frac{\eta \sigma \phi}{\left(\eta+\mu_{1}\right)\left(\phi+\mu_{1}\right)}}{\left(\tau+\varepsilon+\sigma+\mu_{1}\right)}\right)\right)} \\
& \left.R^{*}=\frac{\eta \sigma \lambda^{*} a}{\left(\mu_{1}+\phi\right)\left(\mu_{1}+\eta\right)\left(\tau+\varepsilon+\mu_{1}+\sigma\right)\left(\mu_{1}+\lambda^{*}\left(1-\frac{\eta \sigma \phi}{\left(\eta+\mu_{1}\right)\left(\phi+\mu_{1}\right)}\right)\right.}\right)
\end{aligned}
$$

$\lambda^{*}$ is the solution of the following quadratic polynomial

$$
A\left(\lambda^{*}\right)^{2}+B \lambda^{*}+C=0
$$

where

$$
\begin{gathered}
C=0 \\
A=\left(\mu_{1}+\phi\right)\left(\mu_{1}+\eta\right)\left[\kappa\left(\mu_{2}+\gamma\right)\left(\tau+\varepsilon+\mu_{1}+\sigma\right)-\tau+\frac{\phi \eta \sigma}{\left(\mu_{1}+\phi\right)\left(\mu_{1}+\eta\right)}\right] \\
B=\left(\mu_{1}+\phi\right)\left(\mu_{1}+\eta\right)\left(\tau+\varepsilon+\mu_{1}+\sigma\right)\left[\alpha \delta a-\kappa \mu_{1}\left(\mu_{2}+\gamma\right)\left(\tau+\varepsilon+\mu_{1}+\sigma\right)\right]
\end{gathered}
$$

From Equation (9) it follows that

$$
\lambda^{*}\left(A \lambda^{*}+B\right)=0
$$

implying that

$$
\lambda_{1}^{*}=0
$$

which corresponds to the disease free equilibrium.

$$
\lambda^{*}=-\frac{B}{A} .
$$

This gives

$$
\lambda^{*}=-\frac{\left(\mu_{1}+\phi\right)\left(\mu_{1}+\eta\right)\left(\tau+\varepsilon+\mu_{1}+\sigma\right)\left[\alpha \delta a-\kappa \mu_{1}\left(\mu_{2}+\gamma\right)\left(\tau+\varepsilon+\mu_{1}+\sigma\right)\right]}{\left(\mu_{1}+\phi\right)\left(\mu_{1}+\eta\right)\left[\kappa\left(\mu_{2}+\gamma\right)\left(\tau+\varepsilon+\mu_{1}+\sigma\right)-\tau+\frac{\phi \eta \sigma}{\left(\mu_{1}+\phi\right)\left(\mu_{1}+\eta\right)}\right]} .
$$

It follows that

$$
\lambda^{*}=-\frac{\left(\mu_{1}+\phi\right)\left(\mu_{1}+\eta\right)\left(\tau+\varepsilon+\mu_{1}+\sigma\right)\left[R_{e}-1\right]}{\left(\mu_{1}+\phi\right)\left(\mu_{1}+\eta\right)\left[\kappa\left(\mu_{2}+\gamma\right)\left(\tau+\varepsilon+\mu_{1}+\sigma\right)-\tau+\frac{\phi \eta \sigma}{\left(\mu_{1}+\phi\right)\left(\mu_{1}+\eta\right)}\right]}
$$

where

$$
R_{e}=\frac{\alpha \delta a}{\kappa \mu_{1}\left(\tau+\varepsilon+\mu_{1}+\sigma\right)\left(\mu_{2}+\gamma\right)} .
$$

Corresponding to unique endemic equilibrium $E^{*}$ where $R_{e}>1, B<0$ and $A<0$. From this result we state the following theorem which will be proved by using bifurcation diagram and centre manifold theorem.

Theorem 3 
The unique endemic equilibrium $E^{*}$ exists if $A>0, B<0$ and $R_{e}>1$, and is locally stable if $R_{e}>1$, and unstable if $R_{e}<1$.

\section{Determination of Forward or Backward Bifurcation}

From Equation (9), it follows that there is no backward bifurcation since the value of $C=0$, hence no multiple equilibria. Therefore existence of unique endemic equilibrium which is locally stable for $R_{e}>1$ and unstable if $R_{e}<1$ was explored by a forward bifurcation diagram obtained when a graph of proportion infective population " $I$ " against effective reproduction number " $R_{e}$ " is drawn as shown below.

Figure 2 reveal a forward bifurcation when

$$
\begin{aligned}
& a=5, k=100000, \alpha=1, r=0.2, \\
& \varepsilon=0.015, g=1, \delta=5, \gamma=0.000000001, \\
& \eta=0.95, \phi=0.04, \sigma=0.14, \\
& \mu_{1}=0.0000548, \mu_{2}=2, \mu_{3}=0.000001
\end{aligned}
$$

From Figure 2, the two equilibrium points exchange stability depending on the value of $R_{e}$. A transcriti$\mathrm{cal} /$ forward bifurcation in the equilibrium points occur at $R_{e}=1$. If $R_{e}<1$, no biologically meaningful endemic equilibrium solution exists and the disease free equilibrium is the only local attractor. But if $R_{e}>1$, the endemic equilibrium exists and is the only local attractor while the disease free equilibrium is a saddle point.

The local asymptotic stability of endemic equilibrium will be analysed by using the Centre Manifold theory [9] and it is shown that it is stable under certain conditions. The nonlinear model system (1) shows that it will exhibit a backward bifurcation which occurs at $R_{e}=1$ under certain conditions otherwise it will exhibit a forward bifurcation at $R_{e}=1$ as shown in Figure 2 and is locally stable.

\subsection{Global Stability of the Endemic Equilibrium Point (EEP)}

\section{Theorem 4}

If $R_{e}>1$, the endemic equilibrium $E^{*}$ of the non-linear model (1) is globally asymptotically stable [10].

Proof:

To establish the global stability of the endemic equilibrium $E^{*}$ we construct the following positive Lyapunov function $L$ as follows;

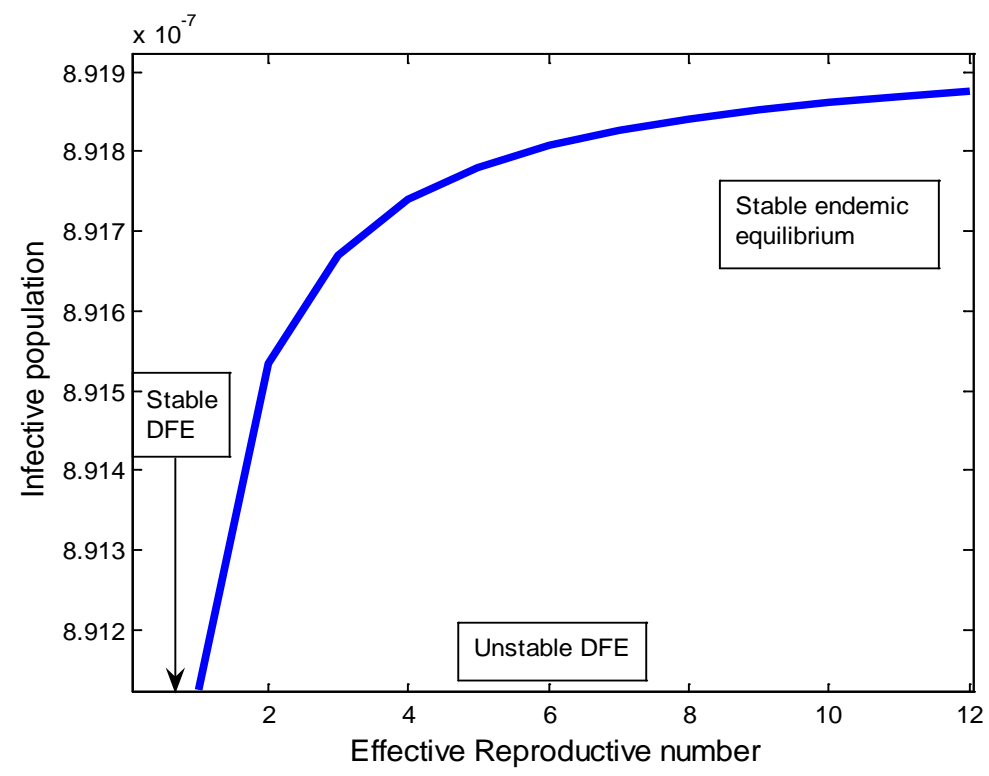

Figure 2. The figure of proportion infective population " $I$ " against effective reproduction number “ $R_{e}$ ”. 


$$
\begin{aligned}
L\left(S^{*}, I^{*}, V^{*}, P^{*}, T^{*}, R^{*}\right)= & \left(S-S^{*}-S^{*} \log \frac{S^{*}}{S}\right)+\left(I-I^{*}-I^{*} \log \frac{I^{*}}{I}\right) \\
& +\left(V-V^{*}-V^{*} \log \frac{V^{*}}{V}\right)+\left(P-P^{*}-P^{*} \log \frac{P^{*}}{P}\right) \\
& +\left(T-T^{*}-T^{*} \log \frac{T^{*}}{T}\right)+\left(R-R^{*}-R^{*} \log \frac{R^{*}}{R}\right)
\end{aligned}
$$

Direct calculation of the derivative of $L$ along the solutions of (1) gives

$$
\begin{aligned}
& \frac{\mathrm{d} L}{\mathrm{~d} t}=\left(\frac{S-S^{*}}{S}\right) \frac{\mathrm{d} S}{\mathrm{~d} t}+\left(\frac{I-I^{*}}{I}\right) \frac{\mathrm{d} I}{\mathrm{~d} t} \\
&+\left(\frac{V-V^{*}}{V}\right) \frac{\mathrm{d} V}{\mathrm{~d} t}+\left(\frac{P-P^{*}}{P}\right) \\
& \frac{\mathrm{d} P}{\mathrm{~d} t}+\left(\frac{T-T^{*}}{T}\right) \frac{\mathrm{d} T}{\mathrm{~d} t}+\left(\frac{R-R^{*}}{R}\right) \frac{\mathrm{d} R}{\mathrm{~d} t}
\end{aligned}
$$

But

$$
\begin{gathered}
\frac{\mathrm{d} S}{\mathrm{~d} t}=a-\frac{\alpha V S}{\kappa+V}-\mu_{1} S+\phi R, \\
\frac{\mathrm{d} V}{\mathrm{~d} t}=g+\delta I-\mu_{2} V-\gamma V, \\
\frac{\mathrm{d} P}{\mathrm{~d} t}=\gamma V-\mu_{3} P, \\
\frac{\mathrm{d} T}{\mathrm{~d} t}=\sigma I-\left(\mu_{1}+\eta\right) T, \\
\frac{\mathrm{d} R}{\mathrm{~d} t}=\eta T-\left(\mu_{1}+\phi\right) R+\tau I,
\end{gathered}
$$

This implies that

$$
\begin{aligned}
\frac{\mathrm{d} L}{\mathrm{~d} t}= & \left(\frac{S-S^{*}}{S}\right)\left(a-\lambda S-\mu_{1} S+\phi R\right) \\
& +\left(\frac{I-I^{*}}{I}\right)\left(\lambda S-\left(\tau+\varepsilon+\mu_{1}+\sigma\right) I\right) \\
& +\left(\frac{V-V^{*}}{V}\right)\left(g+\delta I-\mu_{2} V-\gamma V\right) \\
& +\left(\frac{P-P^{*}}{P}\right)\left(\gamma V-\mu_{3} P\right) \\
& +\left(\frac{T-T^{*}}{T}\right)\left(\sigma I-\left(\mu_{1}+\eta\right) T\right) \\
& +\left(\frac{R-R^{*}}{R}\right)\left(\eta T-\left(\mu_{1}+\phi\right) R+\tau I\right)
\end{aligned}
$$

Substitute

$S=S-S^{*}, I=I-I^{*}, V=V-V^{*}, P=P-P^{*}, T=T-T^{*}$ and $R=R-R^{*}$

Therefore 


$$
\begin{aligned}
\frac{\mathrm{d} L}{\mathrm{~d} t}= & \left(\frac{S-S^{*}}{S}\right)\left(a-\lambda\left(S-S^{*}\right)-\mu_{1}\left(S-S^{*}\right)+\phi\left(R-R^{*}\right)\right) \\
& +\left(\frac{I-I^{*}}{I}\right)\left(\lambda\left(S-S^{*}\right)-\left(\tau+\varepsilon+\mu_{1}+\sigma\right)\left(I-I^{*}\right)\right) \\
& +\left(\frac{V-V^{*}}{V}\right)\left(\mu_{2}-\gamma\right)\left(V-V^{*}\right)+g+\delta\left(I-I^{*}\right) \\
& +\left(\frac{P-P^{*}}{P}\right)\left(\gamma\left(V-V^{*}\right)-\mu_{3}\left(P-P^{*}\right)\right) \\
& +\left(\frac{T-T^{*}}{T}\right)\left(\sigma\left(I-I^{*}\right)-\left(\mu_{1}+\eta\right)\left(T-T^{*}\right)\right) \\
& +\left(\frac{R-R^{*}}{R}\right)\left(\eta\left(T-T^{*}\right)-\left(\mu_{1}+\phi\right)\left(R-R^{*}\right)+\tau\left(I-I^{*}\right)\right)
\end{aligned}
$$

which gives

$$
\begin{aligned}
\frac{\mathrm{d} L}{\mathrm{~d} t}= & \frac{\left(S-S^{*}\right)^{2}}{S}\left(-\lambda-\mu_{1}\right)+\frac{a\left(S-S^{*}\right)}{S}+\frac{\left(S-S^{*}\right)}{I}+\frac{\phi\left(R-R^{*}\right)\left(S-S^{*}\right)}{S} \\
& +\left(\frac{I-I^{*}}{I}\right) \lambda\left(S-S^{*}\right)-\frac{\left(I-I^{*}\right)^{2}}{I}\left(\tau+\varepsilon+\mu_{1}+\sigma\right) \\
& +\frac{\left(V-V^{*}\right)^{2}}{V}\left(\mu_{2}-\gamma\right)+\frac{\left(V-V^{*}\right)\left(I-I^{*}\right) \delta}{V}+\frac{\left(P-P^{*}\right)\left(V-V^{*}\right) \gamma}{P} \\
& -\frac{\mu_{3}\left(P-P^{*}\right)^{2}}{P}+\frac{\left(T-T^{*}\right)\left(I-I^{*}\right) \sigma}{T}-\frac{\left(\mu_{2}+\eta\right)\left(T-T^{*}\right)^{2}}{T} \\
& +\frac{\left(R-R^{*}\right)\left(T-T^{*}\right) \eta}{R}-\frac{\left(\mu_{1}+\phi\right)\left(R-R^{*}\right)^{2}}{R}+\frac{\tau\left(I-I^{*}\right)}{R}
\end{aligned}
$$

Collecting positive and negative terms together in the system (13), we obtain

$$
\frac{\mathrm{d} L}{\mathrm{~d} t}=Q-Z
$$

If we let

$$
\begin{aligned}
Q= & \frac{1}{S}\left(a\left(S-S^{*}\right)+\left(S-S^{*}\right)+\phi\left(R-R^{*}\right)\left(S-S^{*}\right)\right)+\frac{1}{I}\left(\lambda\left(I-I^{*}\right)\left(S-S^{*}\right)\right) \\
& +\frac{1}{V}\left(\left(V-V^{*}\right)^{2}\left(\mu_{2}-\gamma\right)+\left(V-V^{*}\right)\left(I-I^{*}\right) \delta\right)+\frac{1}{P}\left(P-P^{*}\right)\left(V-V^{*}\right) \gamma \\
& +\frac{1}{T}\left(T-T^{*}\right)\left(I-I^{*}\right) \sigma+\frac{1}{R}\left(R-R^{*}\right)\left(T-T^{*}\right) \eta+\tau\left(I-I^{*}\right)
\end{aligned}
$$

And

$$
\begin{aligned}
Z= & \frac{1}{S}\left(S-S^{*}\right)^{2}+\left(\lambda+\mu_{1}\right)+\frac{1}{I}\left(I-I^{*}\right)^{2}\left(\tau+\varepsilon+\mu_{1}+\sigma\right)+\frac{1}{P} \mu_{3}\left(P-P^{*}\right)^{2} \\
& +\frac{1}{T}\left(\mu_{2}+\eta\right)\left(T-T^{*}\right)^{2}+\frac{1}{R}\left(\mu_{1}+\phi\right)\left(R-R^{*}\right)^{2}
\end{aligned}
$$


then $\frac{\mathrm{d} L}{\mathrm{~d} t}<0$ and only if $S=S^{*}, I=I^{*}, V=V^{*}, P=P^{*}, T=T^{*}$ and $R=R^{*}$. Therefore the maximum compact invariant set in $\left\{(S, I, V, P, T, R) \in \Omega: \frac{\mathrm{d} L}{\mathrm{~d} t}=0\right\}$ is the singleton $\left\{E^{*}\right\}$ is the endemic equilibrium of the model system (1). Then by LaSalle's invariant principle it implies that $E^{*}$ is globally asymptotically stable in the interior of $\Omega$ if $Q<Z$ [11].

\subsection{Model in the Absence of Treatment $(\sigma=0$ and $T \rightarrow 0$ )}

Now we consider the situation when there is no treatment of the acute infective i.e. $(\sigma=0)$ and $(T \rightarrow 0)$. We therefore obtain the effective reproduction number as

$$
R_{e_{1}}=\frac{\alpha \delta a}{\kappa \mu_{1}\left(\mu_{2}+\gamma\right)\left(\tau+\varepsilon+\mu_{1}\right)} .
$$

We note that $R_{e}<R_{e_{1}}$ as $R_{e} \rightarrow R_{e_{1}}$ when $\sigma \rightarrow 0$, therefore we conclude that the endemicity of the infection in this case is increased in the absence of treatment.

\subsection{Model in the Absence of Bacteriophage $(P=0$ and $\gamma=0)$}

In this case, we consider the situation where there is no bacteriophage in the model system (1). Since there is no bacteriophage then, $\gamma=0$ implying that $P=0$. We therefore obtain the effective reproduction number as

$$
R_{e_{2}}=\frac{\alpha \delta a}{\kappa \mu_{1}\left(\tau+\varepsilon+\mu_{1}+\sigma\right)\left(\mu_{2}\right)} .
$$

In this situation, it is observed that $R_{e}<R_{e_{1}}<R_{e_{2}}$, when $\gamma=0$ and $R_{e_{1}} \rightarrow R_{e_{2}}$. Therefore we conclude that the infection in this case increases in the absence of bacteriophage which may contribute to lessen the severity of cholera epidemics by reducing the number of Vibrio cholerae in the environment. After analysing the two epidemiological situations discussed above, it may be concluded that in the presence of both bacteriophage and treatment in the model system, the disease tends to the disease free equilibrium points, otherwise the disease tends to endemic state. Therefore the presence of bacteriophage and treatment can reduce the number of Vibrio cholerae in the environment and the number of infectives within the society is also decreased, hence the disease tends to die out.

\section{Numerical Simulation}

In order to illustrate analytical results of the study, numerical simulations of the nonlinear model system (1) are carried out using the set of estimated parameter values below

$$
\begin{aligned}
& a=5, \alpha=5, \tau=0.2, \varepsilon=0.015, \kappa=1000000, \sigma=0.14, \gamma=4, g=1, \\
& \eta=0.97, \phi=0.7, \mu_{1}=0.0000548, \mu_{2}=2, \delta=5, \mu_{3}=0.000001 .
\end{aligned}
$$

Figure 3 shows the proportion of susceptible population plotted against infected population and Vibrio cholerae population. Then the proportion of Bacteriophage population plotted against Vibrio cholerae population. This shows the dynamic behaviour of the endemic of the model system (1) using the parameter values in (15) for different initial starting values in three cases as shown below

1. $S(0)=1500, I(0)=1300, V(0)=1000, P(0)=500, T(0)=1000$ and $R(0)=600$

2. $S(0)=1100, I(0)=800, V(0)=500, P(0)=400, T(0)=300$ and $R(0)=200$

3. $S(0)=1500, I(0)=1200, V(0)=300, P(0)=700, T(0)=1000$ and $R(0)=800$

Figure 4 shows the proportion of treated population plotted against infected and recovered population. This shows the dynamic behaviour of the endemic of the model system (1) using the parameter values in (15) for different initial values in three cases as shown below 

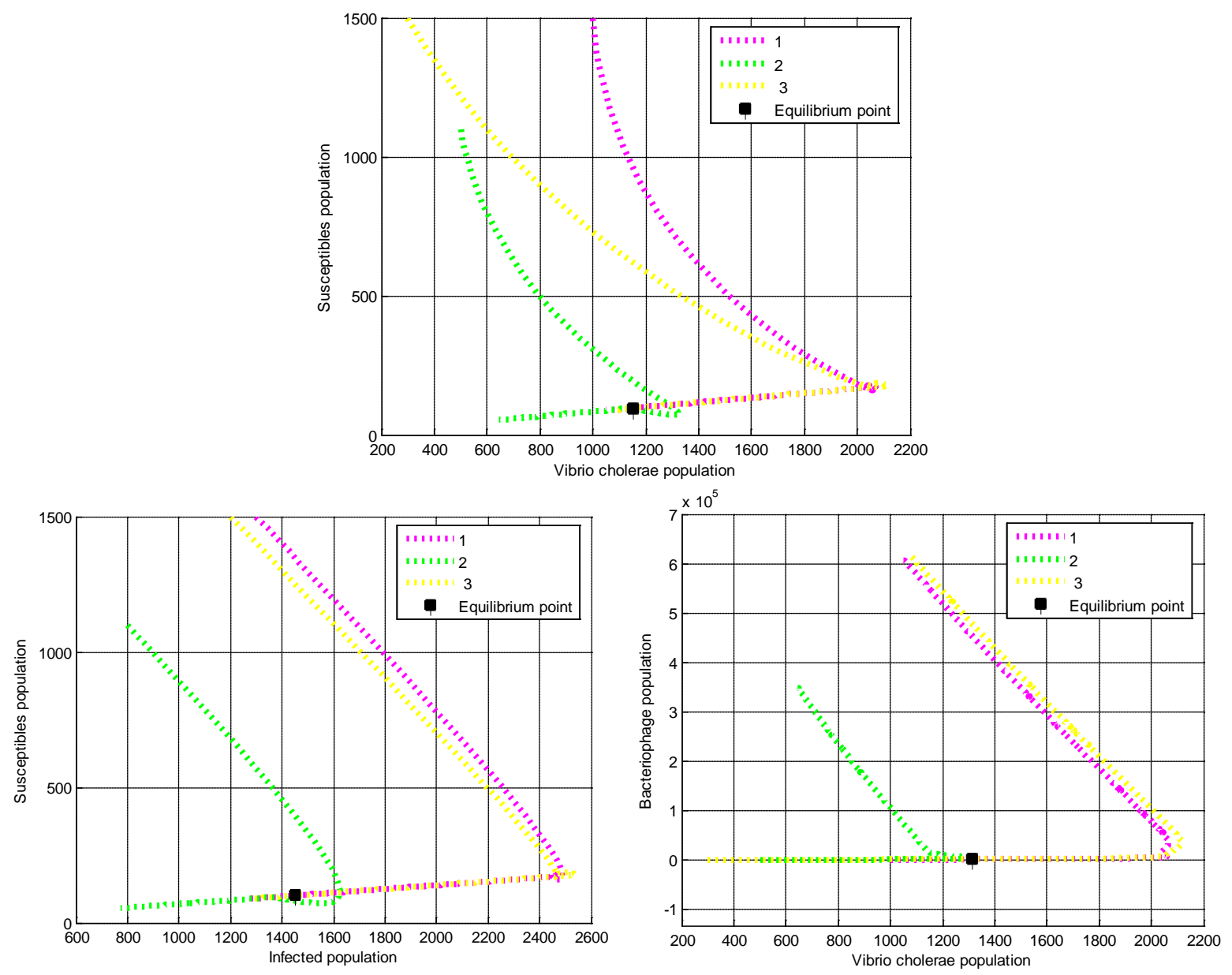

Figure 3. Variation of Proportion of Susceptible Population against Infected Population and Vibrio cholerae Population, and then proportion of Bacteriophage population against Vibrio cholerae population.
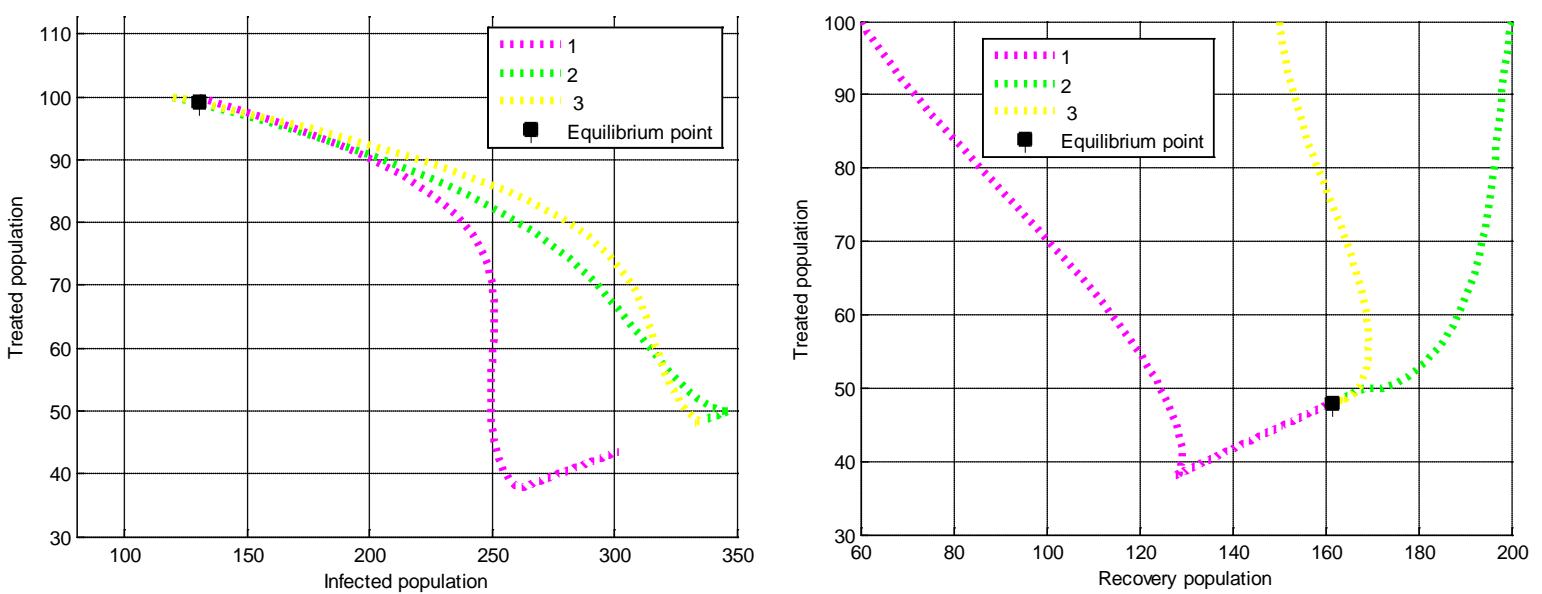

Figure 4. Variation of Proportion of Treated Population against Infected and Recovered population.

1. $S(0)=150, I(0)=130, V(0)=100, P(0)=50, T(0)=100$ and $R(0)=60$

2. $S(0)=200, I(0)=120, V(0)=40, P(0)=80, T(0)=100$ and $R(0)=150$ 
3. $S(0)=170, I(0)=120, V(0)=110, P(0)=80, T(0)=100$ and $R(0)=200$

The equilibrium point of the endemic equilibrium $E^{*}$ was obtained as

$$
\begin{gathered}
S^{*}=100.9 \text { and } I^{*}=1448, S^{*}=96.14 \text { and } V^{*}=1152, P^{*}=1553 \text { and } V^{*}=1315, \\
T^{*}=99.12 \text { and } I^{*}=130.4, T^{*}=99.12 \text { and } R^{*}=130.4
\end{gathered}
$$

It is observed from Figure 3 and Figure 4 that for any starting initial value, the solution curve tend to equilibrium $E^{*}$. Therefore we conclude that the model system (1) is globally stable about this endemic equilibrium $E^{*}$ for the parameters displayed in Equation (15).

Figures 5(a)-(c) show the variation of proportion of total population in different classes, Treated population, Recovery population for different values of $\eta$ (the rate of recovery (perday)).

From Figure 5(a), it is observed that when the bacteriophage population increases continuously, the number of Vibrio cholerae in the system decreases. The number of infectives decreases due to the fact that, the function of bacteriophage is to reduce the number of Vibrio cholerae that causes the disease (Cholera), so when the number of Vibrio cholerae decreases the number of infectives also decreases. Furthermore, from the figure it is observed that the treated population decreases, then move to recovered population and finally to susceptible population

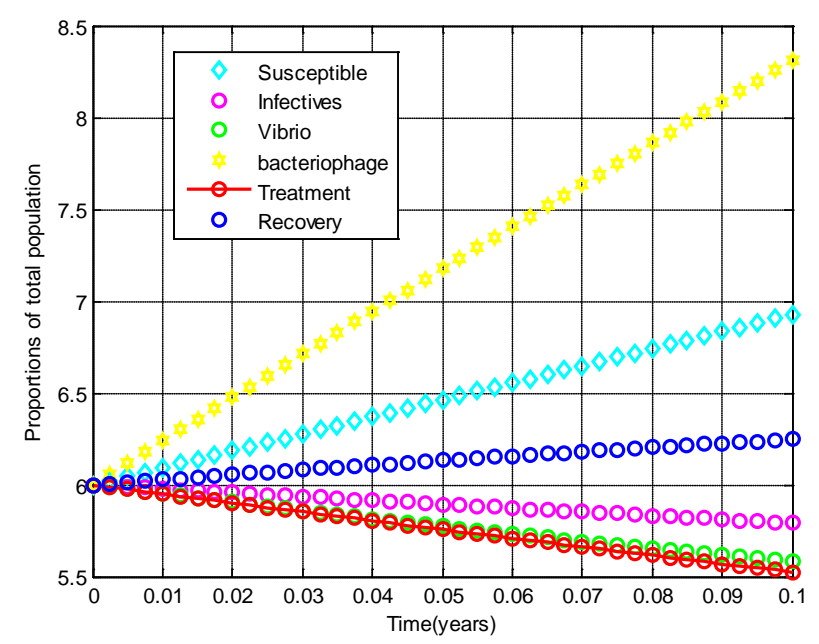

(a)

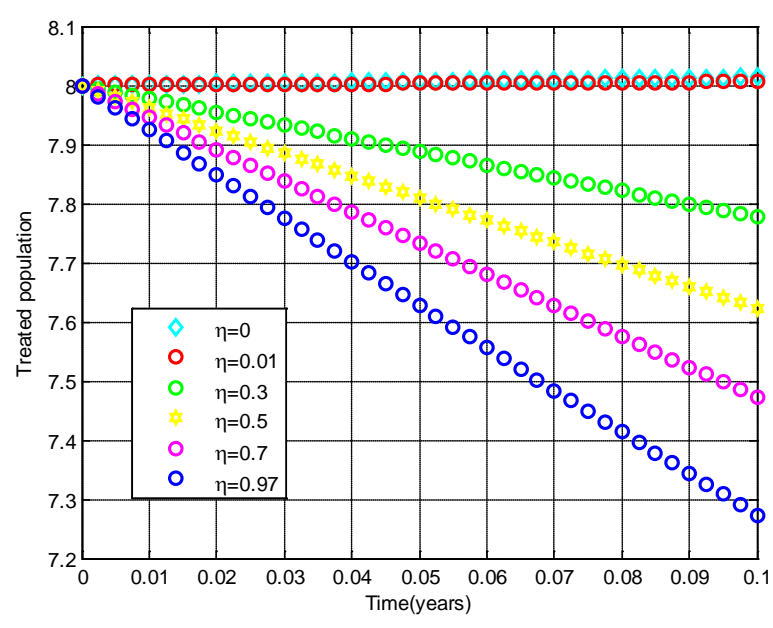

(b)

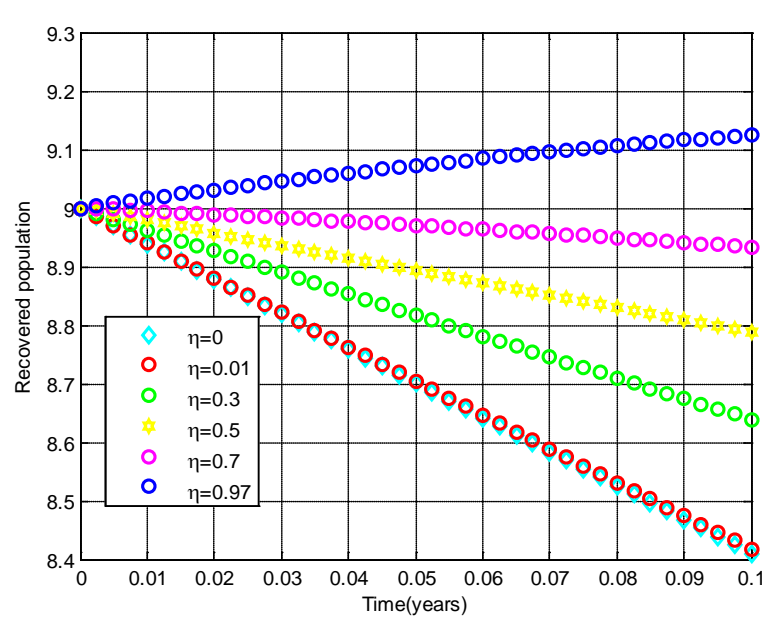

(c)

Figure 5. Distribution of population with time in all classes of human, Vibrio cholerae and Bacteriophage, variation of proportion of treated population, Recovered population for different values of $\eta$ (the rate of recovery (per day)). 
From Figure 5(b), it is observed that, when the value of eta increases the treated population decrease that is due to the fact that many infected people get treatment as a result they recovery and go back to susceptible population, so we can conclude that when the number of eta increase the treated population decrease.

From Figure 5(c), it is observed that the recovered population increases as the number of eta increases. When $(\eta=0)$ the number of recovery population decrease because there is no treatment, but when $(\eta=0.97)$ the recovered population increase due to combination of treatment and bacteriophage in the system.

Figure 6(a) and Figure 6(b) shows the variation of proportion of Vibrio cholerae population for different values of $\gamma$ (phage adsorption rate (per day)) and the variation of proportion of infected population for different values of $\sigma$ (the rate of treatment (per day)).

From Figure 6(a), it is observed that when the rate of treatment increases, the infected population decreases.

From Figure 6(b), it is observed that when the phage absorption rate increases, the Vibrio cholerae population decreases.

\section{Discussion and Conclusion}

A nonlinear mathematical model has been analysed to study the effect of bacteriophage and treatment in the environment while the cholera epidemic is in progress. This study is the extended work done by [6]. Qualitative analysis of the model shows that the model has two equilibrium points, the disease-free equilibrium and endemic equilibrium points. The stabilities of the equilibrium points are investigated. The model shows that the disease-free equilibrium is locally asymptotically stable by using comparison theory at threshold parameter less than unity and unstable at threshold parameter greater than unity, but globally the disease-free equilibrium is not stable due to existence of forward bifurcation at threshold parameter equal to unity. The analysis shows the existence of unique endemic equilibrium that is locally stable when the threshold parameter exceeds unity due to existence of forward bifurcation at threshold parameter equal to unity. Using Lyapunov technique, endemic equilibrium is globally stable under certain conditions. A numerical study of the model was carried out to see the effect of key parameters on the cholera bacteriophage and treatment. The analysis shows that the combination of cholera bacteriophage and treatment has positive impact on the cholera eradication. It is clear and also observed that as the number of bacteriophage increases, the Vibrio cholerae decreases as a result of the infected population decreases. Therefore, bacteriophage may also be used as biological control agent in cholera endemic area. Also it is found that, the recovered population increases as the rate of treatment $\eta$ increases i.e. when $\eta=0$ the number of recovered decreases because there is no treatment, but when $\eta=0.97$ the recovered population increases because the infected population received treatment and become recovery. Therefore the presence of bacteriophage and treatment reduce the number of Vibrio cholerae in the environment and the number of infected population within the

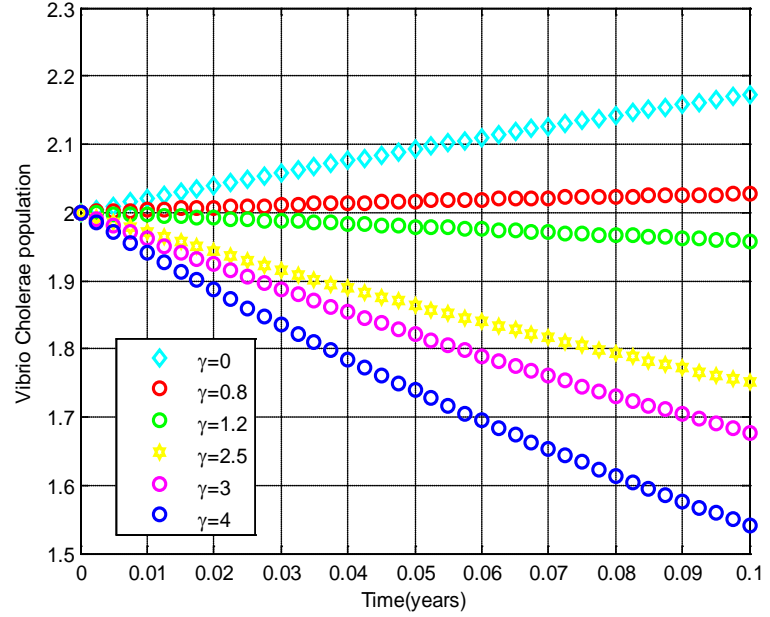

(a)

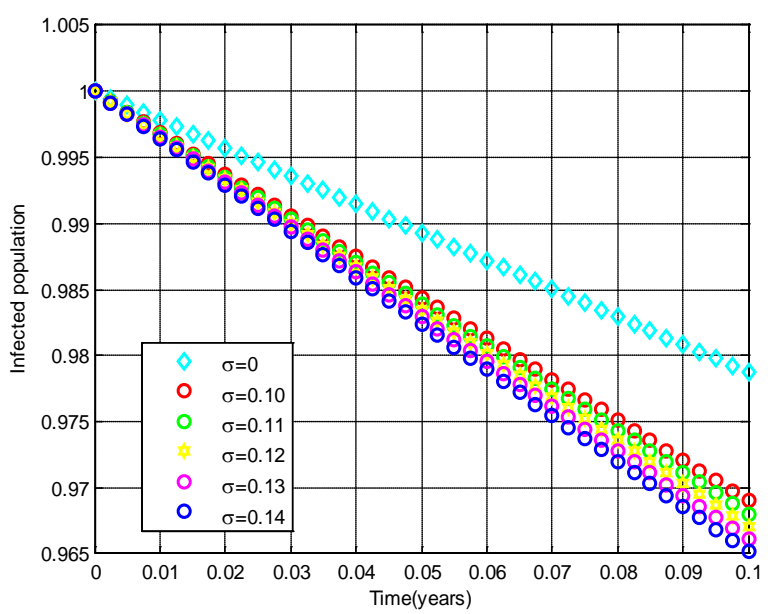

(d)

Figure 6. Shows the variation of proportion of Vibrio cholerae population for different values of $\gamma$ (phage adsorption rate (per day)) and the variation of proportion of infected population for different values of $\sigma$ (the rate of treatment (per day)). 
society is also decreased hence, the disease tends to die out.

Based on the results of this study, we conclude that the most effective way to control cholera epidemic is well achieved by involving both bacteriophage and treatment. However, it is important to note that phage can reduce the number of Vibrio cholerae in the environment. Consequently, number of infected population within the society is also decreased and severity of the disease is also checked. Hence by using phage as a biological control agent in the endemic areas, cholera is self-limiting in nature. Moreover, therapeutic treatment which includes hydration therapy, antibiotics and water sanitation should be administered during the cholera epidemic. Furthermore, people should be educated on the awareness of the effective prevention methods which includes provision and use of clean drinking water, hand washing, environmental hygiene and sanitation, and also avoidance of potentially contaminated foods.

\section{References}

[1] Faruque, S.M., Saha, M.N., Asadulghani, Bag, P.K., Bhadra, R.K., Bhattacharya, S.K., Sack, R.B. and Takeda, Y. (2000) Genomic Diversity among Vibrio cholera O139 Strains Isolated in Bangladesh and India, 1992-1998. FEMS Microbiology Letters, 184, 279-284.

[2] Zuckerman, J.N., Rombo, L. and Fisch, A. (2007) The True Burden and Risk of Cholera Implications for Prevention and Control. The Lancet Infectious Diseases, 7, 521-530. http://dx.doi.org/10.1016/S1473-3099(07)70138-X

[3] Sack, D.A., Sack, R.B., Nair, G.B. and Siddique, A.K. (2004) Cholera. The Lancet, 363, 223-233. http://dx.doi.org/10.1016/S0140-6736(03)15328-7

[4] WHO (2012) Cholera Fact Sheet N 107. http://www.who.int/mediaCentre/factsheets/Fs107/en/index.html

[5] Hertley, D.M., Morris, J.G. and Smith, D.L. (2006) Hiperinfectivity: A Critical Element in the Ability Vibrio cholerae to Cause Epidemics. PLoS Medicine, 3, 63-69.

[6] Das, P. and Mukherjee, D. (2012) Qualitative Analysis of Cholera Bacteriophage Model. International scholarly Research Network Biomathematics, 2012, Article ID: 621939. http://dx.doi.org/10.5402/2012/621939

[7] Bhunu, C.P. and Mushayabasa, S. (2012) Assessing the Impact of Increasing Antimicrobial Resistance of Vibrio cholera on the Future Trends of Cholera Epidemic. International Scholarly Research Network Biomathematics, 2012, Article ID: 127492.

[8] Driessche, P.V. and Watmough, J. (2002) Reproduction Numbers and Sub-Threshold Endemic Equilibria for Compartmental Models of Disease Transmission. Mathematical Biosciences, 180, 29-48. http://dx.doi.org/10.1016/S0025-5564(02)00108-6

[9] Gill, J.J., Sabour, P.M., Leslie, K.E. and Griffiths, M.W. (2006) Bovine Whey Proteins Inhibit the Interaction of Staphylococcus aureus and Bacteriophage. Journal of Applied Microbiology, 101, 377-86. http://dx.doi.org/10.1111/j.1365-2672.2006.02918.x

[10] Ratera, S., Massawe, E.S. and Makinde, O.D. (2012) Modelling the Effect of Screening and Treatment on Transmission of HIV/AIDS Infection in Population. American Journal of Mathematics and Statistics, 2, 75-88.

[11] LaSalle, J.P. (1976) The Stability of Dynamical System. SIAM Review, 21, 418-420. 